\title{
Bidirectional MIMO Channel Tracking Based on PASTd and Performance Evaluation
}

\author{
Livnat Ehrenberg, ${ }^{1}$ Sharon Gannot (EURASIP Member), ${ }^{1}$ Ofer Shayevitz, ${ }^{2}$ \\ Amir Leshem, ${ }^{1}$ and Ephraim Zehavi ${ }^{1}$ \\ ${ }^{1}$ School of Engineering, Bar-Ilan University, Ramat-Gan 52900, Israel \\ 2 Information Theory and Applications Center, University of California, San Diego, CA 92093, USA
}

Correspondence should be addressed to Sharon Gannot, gannot@eng.biu.ac.il

Received 23 September 2009; Revised 25 May 2010; Accepted 28 July 2010

Academic Editor: Tim Davidson

Copyright (C) 2010 Livnat Ehrenberg et al. This is an open access article distributed under the Creative Commons Attribution License, which permits unrestricted use, distribution, and reproduction in any medium, provided the original work is properly cited.

\begin{abstract}
We consider a bidirectional time division duplex (TDD) multiple-input multiple-output (MIMO) communication system with time-varying channel and additive white Gaussian noise (AWGN). A blind bidirectional channel tracking algorithm, based on the projection approximation subspace tracking (PAST) algorithm, is applied in both terminals. The resulting singular value decomposition (SVD) of the channel matrix is then used to approximately diagonalize the channel. The proposed method is applied to an orthogonal frequency-division multiplexing-(OFDM-)MIMO setting with a typical indoor time-domain reflection model. The computational cost of the proposed algorithm, compared with other state-of-the-art algorithms, is relatively small. The Kalman filter is utilized for establishing a benchmark for the obtained performance of the proposed tracking algorithm. The performance degradation relative to a full channel state information (CSI) due to the application of the tracking algorithm is evaluated in terms of average effective rate and the outage probability and compared with alternative tracking algorithms. The obtained results are also compared with a benchmark obtained by the Kalman filter with known input signal and channel characteristics. It is shown that the expected degradation in performance of frequency-domain algorithms (which do not exploit the smooth frequency response of the channel) is only minor compared with time-domain algorithms in a range of reasonable signal-to-noise ratio (SNR) levels. The proposed bidirectional frequency-domain tracking algorithm, proposed in this paper, is shown to attain communication rates close to the benchmark and to outperform a competing algorithm. The paper is concluded by evaluating the proposed blind tracking method in terms of the outage probability and the symbol error rate (SER) versus. SNR for binary phase shift keying (BPSK) and 4-Quadrature amplitude modulation (QAM) constellations.
\end{abstract}

\section{Introduction}

In recent years, MIMO-OFDM schemes have gained increased interest in both theoretical and practical aspects. Paulraj et al. [1] provides a comprehensive overview of the MIMO wireless technology including channel models and performance limits. The IEEE802.11 standard [2] is an example for a TDD communication standard that uses an MIMOOFDM scheme. TDD systems use the same carrier frequency for both the uplink and downlink directions. The channel reciprocity assumption is therefore assumed to be valid; that is, the propagation channel from the $i$ th transmitting antenna to the $j$ th receiving antenna is assumed to be equal to the respective channel in the opposite direction. Channel diagonalization is a common practice in MIMO-OFDM communications since it enables better use of the channel degrees of freedom with reduced computational burden. The diagonalization is usually obtained by virtue of SVD of the estimated communication channel. The performance of the system is sensitive to the accuracy of the estimation of the CSI at both the transmitter and the receiver. Due to the timevarying nature of the communication channel and the delay imposed by the TDD regime, channel tracking procedures are called upon [3].

Recently, several channel tracking methods were proposed. A pilot-based estimation procedure (and hence 
nonblind) is proposed by Lebrun et al. [3]. An additional correction stage is incorporated into the SVD of the communication channel to compensate for outdated estimates of the CSI. The proposed architecture is capable of maintaining the channel capacity without using additional pilots. However, no attempt to directly track the computationally intensive SVD was made.

A family of tracking solutions based on the celebrated Kalman filter is next introduced. Komninakis et al. [4] use the Kalman filter in the time domain to track the channel coefficients. The unknown input signals are replaced by the output of a decision feedback equalizer (DFE).

Roman et al. [5] also use the time-domain Kalman tracking, but the DFE operates in the frequency domain. Bulumulla et al. [6] replace the time-domain formulation by its frequency-domain counterpart, exploiting the channel smooth frequency response. Cheng and Dahlhaus [7] compare a time-domain scheme, similar to [4], and a frequency-domain scheme, similar to [6], and show their equivalence. Hou et al. [8] showed that the obtainable mean square error (MSE) of time- and frequency-domain channel estimation procedures is equivalent. Their analysis (based on pilot submission) is only applicable if an appropriate frequency-domain smoothing is performed. The length of the channel in time domain is exploited to perform the smoothing. Haeb-Umbach and Bevermeier [9] use timedomain Kalman filtering (during the preamble) for the initialization of frequency-domain Wiener filter, used for blindly tracking the channel impulse response. Grolleau et al. [10] improved the traditional pilot-based frequency-domain channel estimation, by applying a Kalman filter to the noisy estimates.

All the above tracking algorithms are applied to the channel coefficients (either in time domain or in frequency domain or in both) and are not applicable to direct tracking of the SVD of the channel. Moreover, the Kalman filter is known to impose high computational burden, mainly due to the matrix inversion involved in the procedure.

Dahl et al. [11-13] use an elegant solution for tracking the SVD of the channel. Their blind iterative MIMO algorithm (BIMA) method uses the well-known algebraic power method. It is shown that by exploiting the bidirectional communications, it is possible to iteratively converge to the desired singular vectors of the channel (which correspond to the largest singular values) in both terminals. However, convergence to orthonormal basis vectors is not guaranteed by the BIMA method, and hence another step, involving an application of Orthogonal Triangular Decomposition (QRD) to the obtained singular vectors, is mandatory. The QRD is a computationally demanding procedure. Furthermore, a crucial component of the algorithm is a detecting stage performed by a slicer, which is prone to error propagation problems.

Estimating performance bounds for time-varying channels is a cumbersome task. Time-varying single-input singleoutput (SISO) channel is addressed by Barbarossa and Scaglione [14]. They claim that physical parameters governing the time variation of the channel impulse response, for example, reflection coefficients, delays, and Doppler shifts, vary slowly in time. Based on this claim, they derive a Cramér-Rao lower bound for the parameters as a function of the frame length. Their derivation cannot be directly applied to the more complicated MIMO scenario.

Our aim in the current contribution is threefold. We first derive a bidirectional channel tracking procedure based on the projection approximation subspace tracking deflation (PASTd) algorithm [15]. The procedure is capable of estimating communication channels under TDD regime. A preliminary study of this algorithm was presented in [16]. Later, Liu et al. [17] performed a comprehensive experimental study of the same algorithm. Second, we establish benchmarks, based on the Kalman filter, for the tracking algorithm. The Kalman filter is used in the paper solely as a performance benchmark for our proposed method. Specifically, we compare time- and frequencydomain schemes assuming that the transmitted data is known. We then extend a preliminary study of the Kalman filter as a performance benchmark [18] to deal with bidirectional communications. We show that frequency-domain tracking algorithms, which do not exploit the smooth channel frequency response, are inferior to the computationally more expensive, time-domain tracking schemes. The derived Kalman-based bidirectional frequency-domain benchmark is compared with the proposed method. It is shown that the performance degradation due to the application of the frequency-domain PASTd algorithm is only minor in comparison with the derived benchmark. This study therefore justifies the use of our novel and efficient tracking algorithm. Third, performance measures based on information theoretic notions such as effective communication rate and outage probability are derived and presented for both Gaussian and BPSK data streams. Simulations using an autoregressive (AR) channel model (that comply with Saleh and Valenzuela model [19]) are performed, and the expected performance degradation due to the estimation errors is evaluated.

The structure of this paper is as follows. In Section 2, we state the problem both in the time- and frequencydomains. In Section 3, we derive a state-space representation of the problem at hand and motivate the use of the Kalman filter as a performance benchmark for the channel tracking algorithm. Appendix A is dedicated to the description of the Kalman filter-based benchmark. The proposed bidirectional frequency-domain channel tracking algorithm, which applies the PASTd procedure, is derived in Section 4. Performance measures of the estimated channel matrix, based on channel capacity notions, are discussed in Section 5. Finally, a comprehensive experimental study is performed in Section 6. It is first shown that timedomain schemes are superior to frequency-domain schemes, although comparable performance measures are exhibited in a wide range of SNR levels. Then, the tracking ability and the obtainable communication rate are evaluated and compared with the BIMA method proposed in [12]. Finally, the proposed blind tracking method is evaluated by testing the outage probability and the SER versus SNR for BPSK and 4-QAM constellations. We draw final conclusions in Section 7. 


\section{Problem Statement}

In this section, the MIMO channel in the OFDM framework is presented. We address a bidirectional MIMO channel. We define the communication system from the base station point of view. Thus, the link from the base station to the subscriber will be denoted downlink, and the reverse side will be denoted uplink. We start by formulating the problem in the time-domain and proceed to formulating the frequency domain and the bidirectional frequency domain. This section is concluded by modeling the stochastic process governing the channel time evolution. This model will be used both in the derivation of the Kalman filter benchmark and in the simulations described in the experimental study section.

2.1. Time Domain. Let $N$ denote the OFDM symbol length. Assume that the number of transmitting antennas is $N_{t}$, and the number of the receiving antennas is $N_{r}$. Each of the $N_{t} \times N_{r}$ MIMO channels is assumed to be fading channel with $L$ coefficients $h^{i j}(\ell, n)$, where $1 \leq i \leq N_{t}$ are the transmit antenna indexes, $1 \leq j \leq N_{r}$ are the receiving antenna indexes, and $\ell=0, \ldots, L-1$ are the coefficients indexes. We assume the filters to be time varying, and hence, their values depend on $n$, the symbol index. However, the channels are assumed to be time invariant within the OFDM symbol.

The $i$ th antenna in the base station transmits a sequence of OFDM symbols $x^{i}(m, n)$, where $-\infty<n<\infty$ is the symbol index, and $m=0, \ldots, N-1$ denotes the time index within the OFDM symbol. For the OFDM assumption to hold, each symbol of the transmitted signal consists of data samples preceded by a cyclic prefix (CP). The length of the CP should be greater than or equal to the channel spread $L$, to guarantee proper operation of the OFDM system.

The received signal at the $j$ th antenna of the subscriber is thus a linear combination of transmitted sequences:

$$
y^{j}(m, n)=\sum_{i=1}^{N_{t}} \sum_{\ell=0}^{L-1} h^{i j}(\ell, n) x^{i}(m-\ell, n)+z^{j}(m, n),
$$

where the additive term $z^{j}(m, n)$ is assumed to be temporarily white complex Gaussian noise. Due to the CP, $x^{i}(m-$ $\ell, n)=x^{i}(\bmod (m-\ell, N), n)$. The received signals can be compactly described in a matrix notation:

$$
\mathbf{y}_{n}=\left(\mathbf{I}_{N_{r}} \bigotimes \mathbf{X}_{n}\right) \mathbf{h}_{n}+\mathbf{z}_{n}
$$

where $\otimes$ is the Kronecker product and

$$
\begin{aligned}
\mathbf{h}_{n}^{i j} \triangleq\left[h^{i j}(0, n), h^{i j}(1, n), \ldots, h^{i j}(L-1, n)\right]^{T}, \\
\mathbf{h}_{n} \triangleq\left[\left(\mathbf{h}_{n}^{11}\right)^{T} \ldots\left(\mathbf{h}_{n}^{N_{t} 1}\right)^{T} \ldots\left(\mathbf{h}_{n}^{1 N_{r}}\right)^{T} \ldots\left(\mathbf{h}_{n}^{N_{t} N_{r}}\right)^{T}\right]^{T},
\end{aligned}
$$

$$
\begin{aligned}
& \mathbf{y}_{n} \triangleq \\
& {\left[y^{1}(0, n) \ldots y^{1}(N-1, n) \ldots y^{N_{r}}(0, n) \ldots y^{N_{r}}(N-1, n)\right]^{T},} \\
& \mathbf{z}_{n} \triangleq \\
& {\left[z^{1}(0, n) \ldots z^{1}(N-1, n) \ldots z^{N_{r}}(0, n) \ldots z^{N_{r}}(N-1, n)\right]^{T},} \\
& \mathbf{X}_{n}^{i} \triangleq\left[\begin{array}{ccc}
x^{i}(0, n) & \ldots & x^{i}(-L+1, n) \\
x^{i}(1, n) & \ldots & x^{i}(-L+2, n) \\
& \vdots & \\
x^{i}(N-1, n) & \ldots & x^{i}(N-L, n)
\end{array}\right]_{N \times L}, \\
& \mathbf{X}_{n} \triangleq\left[\begin{array}{llll}
X_{n}^{1} & X_{n}^{2} & \ldots & X_{n}^{N_{t}}
\end{array}\right]_{N \times\left(L N_{t}\right)} \text {. }
\end{aligned}
$$

$\mathbf{z}_{n}$, consisting of concatenated noise samples, is a zeromean, temporarily white, circularly symmetric complex, Gaussian-distributed random vector, $\mathbf{z}_{n} \sim \mathcal{C N}\left(0, \mathbf{R}_{n}\right)$, where $\mathbf{R}_{n} \in \mathbb{C}^{\left(N_{r} N\right) \times\left(N_{r} N\right)}$ is the time-domain noise covariance matrix. If the measurement noise is also spatially white; that is, the noise signals received by the antennas are uncorrelated, the covariance matrix $\mathbf{R}_{n}$ becomes diagonal.

2.2. Frequency Domain. If the CP is larger than or equal to the channel length $L$, the channel effect is converted into a circular convolution, and hence can be conveniently expressed as a multiplication in the frequency domain. Let

$$
y^{j}(\omega, n)=\sum_{m=0}^{N-1} y^{j}(m, n) e^{-j \omega m},
$$

and

$$
h^{i j}(\omega, n) \triangleq \sum_{l=0}^{L-1} h^{i j}(\ell, n) \mathrm{e}^{-j \omega \ell}
$$

where $\omega$ is the frequency bin. Then

$$
\mathbf{y}_{n}(\omega)=\mathbf{H}_{n}(\omega) \mathbf{x}_{n}(\omega)+\mathbf{z}_{n}(\omega)
$$

where

$$
\begin{aligned}
& \mathbf{y}_{n}(\omega) \triangleq\left[\begin{array}{lll}
y^{1}(\omega, n) & \ldots & y^{N_{r}}(\omega, n)
\end{array}\right]^{T}, \\
& \mathbf{x}_{n}(\omega) \triangleq\left[\begin{array}{lll}
x^{1}(\omega, n) & \ldots & x^{N_{t}}(\omega, n)
\end{array}\right]^{T}, \\
& \mathbf{z}_{n}(\omega) \triangleq\left[\begin{array}{lll}
z^{1}(\omega, n) & \ldots & z^{N_{r}}(\omega, n)
\end{array}\right]^{T},
\end{aligned}
$$

and the channel matrix $\mathbf{H}_{n}(\omega)$ is given by

$$
\mathbf{H}_{n}(\omega) \triangleq\left[\begin{array}{ccc}
h^{11}(\omega, n) & \cdots & h^{N_{t} 1}(\omega, n) \\
\vdots & \vdots & \\
h^{1 N_{r}}(\omega, n) & \cdots & h^{N_{t} N_{r}}(\omega, n)
\end{array}\right] .
$$


$\mathbf{z}_{n}(\omega)$ is a circular symmetric complex $\operatorname{AWGN~} \mathbf{z}_{n}(\omega) \sim$ $\mathcal{C} N\left(0, \mathbf{R}_{n}(\omega)\right) . \mathbf{R}_{n} \in \mathbb{C}^{N_{r} \times N_{r}}$ is the frequency-domain noise covariance matrix. In the frequency-domain formulation, the frequency bins are decoupled, hence yielding instantaneous rather than convolutive mixing.

2.3. Bidirectional MIMO System. We consider a bidirectional link, and assume that the gains of the radio frequency (RF) chains on both terminals are fully compensated for so that the channel matrices in both directions reflect only the effect of the physical link itself. Under this reciprocity assumption, the uplink channel model is given by

$$
\tilde{\mathbf{y}}_{n}(\omega)=\mathbf{H}_{n}^{T}(\omega) \widetilde{\mathbf{x}}_{n}(\omega)+\widetilde{\mathbf{z}}_{n}(\omega),
$$

where $\widetilde{\mathbf{x}}_{n}(\omega), \widetilde{\mathbf{z}}_{n}(\omega)$, and $\tilde{\mathbf{y}}_{n}(\omega)$ are the respective subscriber transmitted signals, noise signals, and base station received signals, defined in accordance with the downlink definitions.

For the sake of brevity, we will omit the frequency bin index $\omega$ whenever no confusion can occur. The time-varying downlink channel matrix $\mathbf{H}_{n}$ can be factored using the SVD as follows:

$$
\mathbf{H}_{n}=\mathbf{U}_{n} \mathbf{S}_{n} \mathbf{V}_{n}^{H}
$$

where $\mathbf{U}_{n}^{N_{r} \times N_{r}}$ and $\mathbf{V}_{n}^{N_{t} \times N_{t}}$ are unitary matrices of the left and right singular vectors of $\mathbf{H}_{n}$, respectively, and $H$ denotes the Hermitian (conjugate-transposed) operation. $\mathbf{S}_{n}^{N_{r} \times N_{t}}$ is a diagonal matrix with nonnegative $\left\{s_{n}^{k}\right\}_{k=1}^{\min \left(N_{r}, N_{t}\right)}$ singular values on its main diagonal. Consequently, the SVD factorization of $\mathbf{H}_{n}^{T}$ is given by

$$
\mathbf{H}_{n}^{T}=\mathbf{V}_{n}^{*} \mathbf{S}_{n}^{T} \mathbf{U}_{n}^{T}
$$

Using (10) and (13), the downlink and uplink channels can be rewritten as

$$
\begin{aligned}
& \mathbf{y}_{n}=\mathbf{U}_{n} \mathbf{S}_{n} \mathbf{V}_{n}^{H} \mathbf{x}_{n}+\mathbf{z}_{n}, \\
& \tilde{\mathbf{y}}_{n}=\mathbf{V}_{n}^{*} \mathbf{S}_{n}^{T} \mathbf{U}_{n}^{T} \widetilde{\mathbf{x}}_{n}+\widetilde{\mathbf{z}}_{n} .
\end{aligned}
$$

As a consequence, using $\mathbf{V}_{n}$ as a precoder at the transmission side of the base station and $\mathbf{U}_{n}^{H}$ at the receiving side of the subscriber, and $\mathbf{U}_{n}^{*}$ and $\mathbf{V}_{n}^{T}$ at the respective uplink channel, results in a diagonal channel in both directions.

$$
\begin{aligned}
& \overline{\mathbf{x}}_{n}=\mathbf{V}_{n} \mathbf{x}_{n}, \quad \overline{\mathbf{y}}_{n}=\mathbf{U}_{n} \mathbf{S}_{n} \mathbf{V}_{n}^{H} \overline{\mathbf{x}}_{n}+\mathbf{z}_{n}, \\
& \boldsymbol{\zeta}_{n}=\mathbf{U}_{n}^{H} \overline{\mathbf{y}}_{n} \Longrightarrow \boldsymbol{\zeta}_{n}=\mathbf{S}_{n} \mathbf{x}_{n}+\mathbf{U}_{n}^{H} \mathbf{z}_{n} \\
& \overline{\widetilde{\mathbf{x}}}_{n}=\mathbf{U}_{n}^{*} \tilde{\mathbf{x}}_{n}, \quad \overline{\tilde{\mathbf{y}}}_{n}=\mathbf{V}_{n}^{*} \mathbf{S}_{n}^{T} \mathbf{U}_{n}^{T} \overline{\tilde{\mathbf{x}}}_{n}+\widetilde{\mathbf{z}}_{n}, \\
& \tilde{\boldsymbol{\zeta}}_{n}=\mathbf{V}_{n}^{T} \overline{\tilde{\mathbf{y}}}_{n} \Longrightarrow \tilde{\boldsymbol{\zeta}}_{n}=\mathbf{S}_{n}^{T} \tilde{\mathbf{x}}_{n}+\mathbf{V}_{n}^{T} \widetilde{\mathbf{z}}_{n} .
\end{aligned}
$$

Note that it is sufficient to know $\mathbf{V}_{n}$ at the base station site, and $\mathbf{U}_{n}$ at the subscriber site, for conducting the communication. Note also that since $\mathbf{U}_{n}, \mathbf{V}_{n}$ are unitary matrices, the power constraint is not violated. Now, if the singular values are known, communications in both directions simplify to parallel independent AWGN channels. The bidirectional MIMO system with precoding and postcoding is depicted in Figure 1. The tracking algorithm blocks depicted in Figure 1 are discussed in detail in Section 4.

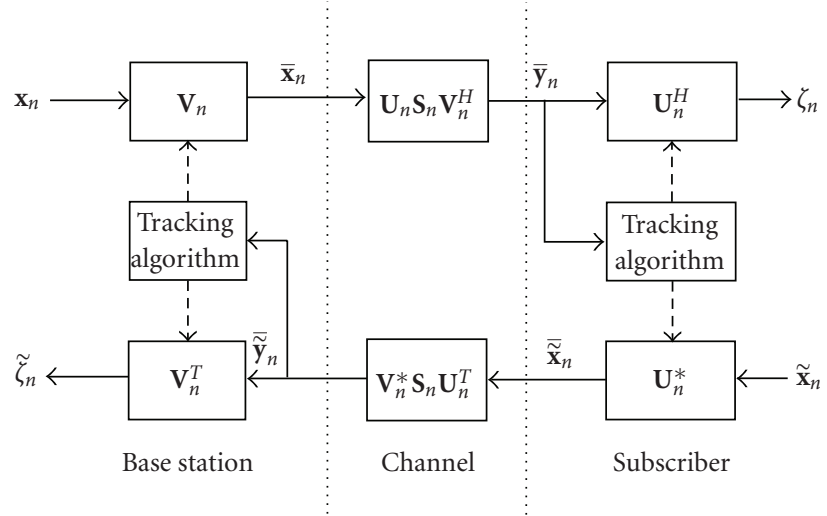

FIgUre 1: Bidirectional MIMO System.

2.4. Channel Model. In many cases the channel coefficients trajectory can be approximately modeled as an autoregressive (AR) process of order $P$. Hence, the $\ell$ th tap of the $i j$ th time-varying channel is given by

$$
h^{i j}(\ell, n)=\sum_{p=1}^{P} \alpha_{\ell}^{i j}(p) h^{i j}(\ell, n-p)+w^{i j}(\ell, n) .
$$

We assume that the frame-dependent filter coefficients can be modeled as a wide-sense stationary (WSS) $\operatorname{AR}(P)$ process. Hence, the driving noise signals $w^{i j}(\ell, n)$ are independently identically distributed (i.i.d.). We further assume that all $N_{t} \times$ $N_{r} \times L$ processes $w^{i j}(\ell, n)$ are uncorrelated.

The time propagation of outdoor fading channels is often modeled as AR processes [6]. The AR coefficients $\alpha_{\ell}^{i j}(p)$ and the variance of the innovation process $w^{i j}(\ell, n)$ can be determined in advance by solving the Yule-Walker equations using the channel correlation matrix. Following Komninakis [4], we assume that the mean of all channel coefficients is zero, and that their autocorrelation sequences are governed by the Doppler shift $f_{D}$

$$
\mathbb{E}\left\{h^{i j}(\ell, n)\left(h^{i j}(\ell, n-m)\right)^{*}\right\} \sim J_{0}\left(2 \Pi f_{D} m T\right),
$$

where $T$ is the duration of a transmitted symbol, $T=1 / \Delta f$. $\Delta f$ is the frequency spacing between the OFDM subcarriers. It was empirically shown that AR modeling fits the trajectory of measured indoor channels as well [16]. Each reflection undergoes a different geographic route. However, we assume that the underlying statistics governing all reflections is identical. It is therefore assumed that the forgetting factor is independent of the coefficient index, that is, $\alpha_{\ell}^{i j}(p)=$ $\alpha^{i j}(p)$, for all $p=1, \ldots, P$. For the clarity of the exposition, we will assume that the order of all AR processes is $P=1$.

\section{State-Space Modeling and Algorithm Benchmark}

In Section 2, the bidirectional MIMO channel model was presented. In Section 4, a tracking scheme, based on the 
channel matrix eigenanalysis, will be derived. The aim of the current section is to motivate the derivation of a benchmark for evaluating the proposed algorithm. Calculating the ability of algorithms to track the time-varying channel coefficients might be a cumbersome task. To simplify the derivation, we propose to use the Bayesian framework. We adopt the celebrated Kalman filter [20], with known channel statistics and input signal, as a method for evaluating the obtainable performance of tracking algorithms, regardless of the particular algorithm used. Obviously, this information is unavailable, restricting the application of the described Kalman filter procedure to merely a performance benchmark. Three instances of the Kalman filter are used in the paper. The time-domain and frequency-domain instances are used for roughly estimating the expected performance loss due to the application of the PASTd-based tracking algorithm in the frequency domain. The bidirectional frequency-domain instance of the Kalman filter is used for evaluating the proposed communication scheme (based on either PASTd or BIMA channel tracking algorithms). The actual application of the Kalman filter as a benchmark for the tracking algorithm is given in Appendix A. The reader is referred to (A.1)-(A.2) for the definition of the state-space model and to Algorithm 2 for the Kalman filter propagation and update stages. In the current section, the state-space formulation of the problem, required for the application of the Kalman filter, is described. This compact formulation is a widely used presentation of dynamic systems. Eventually, in Section 6, the proposed algorithm is evaluated, and its performance is compared with another blind channel tracking algorithm [12] and with the Kalman filter benchmark.

3.1. State-Space Formulation in the Time Domain. In the time-domain formulation, we can identify the desired statevector $\mathbf{d}_{n}$ as the channel vector $\mathbf{h}_{n} \in \mathbb{C}^{\left(L N_{t} N_{r}\right) \times 1}$. Hence, (2) can be immediately identified as a proper measurement equation

$$
\mathbf{y}_{n}=\left(\mathbf{I}_{N_{r}} \bigotimes \mathbf{X}_{n}\right) \mathbf{d}_{n}+\mathbf{z}_{n}
$$

That is $\mathbf{I}_{N_{r}} \otimes \mathbf{X}_{n}$ in (2) can be identified as $\mathbf{G}_{n}$ in (A.2), the measurement matrix (for the time-domain presentation). Define $\mathbf{w}_{n} \in \mathbb{C}^{\left(L N_{t} N_{r}\right) \times 1}$, the innovation vector

$$
\begin{aligned}
& \mathbf{w}_{n}^{i j} \triangleq\left[\begin{array}{llll}
w^{i j}(0, n) & w^{i j}(1, n) & \ldots & w^{i j}(L-1, n)
\end{array}\right]^{T},
\end{aligned}
$$

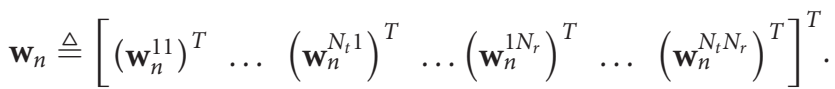

The driving noise $\mathbf{w}_{n}$ is a zero-mean, i.i.d. process with dimensions $L \times N_{t} \times N_{r}$. Hence, its covariance matrix fulfills $\mathbb{E}\left\{\mathbf{w}_{n} \mathbf{w}_{m}^{H}\right\} \triangleq \mathbf{Q}_{n} \delta_{m, n}$. Due to the assumption that all driving noise processes are uncorrelated, $\mathbf{Q}_{n}$ is a diagonal matrix. We further assume that the coefficients obey a decaying power profile. $\mathbf{z}_{n}$ is an $N \times N_{r}$ sensor noise vector defined in (6) with $\mathbb{E}\left\{\mathbf{z}_{n} \mathbf{z}_{n}^{H}\right\}=\mathbf{R}_{n} \delta_{m n}$. The transition matrix of the $\operatorname{AR}(1)$ process, defined in (A.1) $\boldsymbol{\Phi}_{n} \in \mathbb{C}^{\left(L N_{t} N_{r}\right) \times\left(L N_{t} N_{r}\right)}$ is given by

$$
\boldsymbol{\Phi}_{n} \triangleq \operatorname{diag}\left(\left[\left(\boldsymbol{\alpha}^{11}\right)^{T} \ldots\left(\boldsymbol{\alpha}^{N_{t} 1}\right)^{T} \ldots\left(\boldsymbol{\alpha}^{N_{t} N_{r}}\right)^{T}\right]\right),
$$

where the $L \times 1$ vector $\boldsymbol{\alpha}^{i j}$ is defined as

$$
\boldsymbol{\alpha}^{i j} \triangleq\left[\begin{array}{lll}
\alpha^{i j} & \ldots & \alpha^{i j}
\end{array}\right]^{T} .
$$

The state-space formulation in the time domain is summarized in Algorithm 2.

3.2. State-Space Formulation in the Frequency Domain. We derive now the state-space formulation in the frequency domain for either the channel matrix $\mathbf{H}_{n}(\omega)$ or the singular vectors matrices $\mathbf{U}_{n}(\omega)$ and $\mathbf{V}_{n}(\omega)$ for the bidirectional system. As tracking is performed independently for each tone, the spectral smoothness of the channels cannot be exploited.

3.2.1. Tracking the Channel Matrix $H_{n}(\omega)$. An AR(1) model for the matrix $\mathbf{H}_{n}(\omega)$ in the frequency domain can be derived from the time-domain model described in (18).

$$
\begin{aligned}
h^{i j}(\omega, n) & =\sum_{l=0}^{L-1} h^{i j}(\ell, n) e^{-j \omega \ell} \\
& =\sum_{l=0}^{L-1}\left(\alpha^{i j} h^{i j}(\ell, n-1)+w^{i j}(\ell, n)\right) e^{-j \omega \ell} \\
& =\alpha^{i j} h^{i j}(\omega, n-1)+\sum_{l=0}^{L-1} w^{i j}(\ell, n) e^{-j \omega \ell} .
\end{aligned}
$$

Collecting terms, we have

$$
h^{i j}(\omega, n)=\alpha^{i j} h^{i j}(\omega, n-1)+w^{i j}(\omega, n) .
$$

Hence, $h^{i j}(\omega, n)$ is an $\operatorname{AR}(1)$ model as well, with the same coefficients $\left\{\alpha^{i j}\right\}$ (The same result also applies for $\operatorname{AR}(\mathrm{P})$ processes. The proof follows the same lines.). Since the frequency-domain driving noise sequences $w^{i j}(\omega, n)$ are the discrete time Fourier transform (DTFT) of the respective time-domain driving noise sequences $w^{i j}(\ell, n)$, they are also i.i.d. sequences. The independence of the channels also implies that $w^{i j}(\omega, n)$ are independent for different $i j$ pairs. The state-space formulation can now be stated. Using (10) and the formula [21]

$$
\operatorname{vec}(\mathbf{R F T})=\left(\mathbf{F}^{T} \bigotimes \mathbf{R}\right) \operatorname{vec}(\mathbf{T})
$$

we get the measurement equation

$$
\mathbf{y}_{n}(\omega)=\left(\mathbf{I}_{N r} \bigotimes \mathbf{x}_{n}^{T}(\omega)\right) \mathbf{d}_{n}(\omega)+\mathbf{z}_{n}(\omega)
$$

where from (12),

$$
\begin{aligned}
& \mathbf{h}_{n}(\omega) \\
& \triangleq \operatorname{vec}\left(\mathbf{H}_{n}^{T}(\omega)\right) \\
& =\left[h^{11}(\omega, n) \cdots h^{N_{t}}(\omega, n) \cdots h^{1 N r}(\omega, n) \cdots h^{N_{t} N r}(\omega, n)\right]^{T} .
\end{aligned}
$$


Hence, $\mathbf{d}_{n}(\omega) \in \mathbb{C}^{\left(N_{t} N_{r}\right) \times 1}$ can be identified as $\mathbf{h}_{n}(\omega)$, and $\left(\mathbf{I}_{N_{r}} \otimes \mathbf{x}_{n}^{T}(\omega)\right)$ can be identified as the frequency domain measurement matrix $\mathbf{G}_{n}(\omega)$. Further, defining the innovation vector

$$
\mathbf{w}_{n}(\omega) \triangleq\left[\begin{array}{llll}
w^{11}(\omega, n) & \ldots & \ldots & w^{N_{t} N_{r}}(\omega, n)
\end{array}\right]^{T},
$$

and the transition matrix

$$
\boldsymbol{\Phi}_{n} \triangleq \operatorname{diag}\left(\left[\alpha^{11} \cdots \alpha^{N_{t} 1} \cdots \alpha^{N_{t} N_{r}}\right]\right),
$$

the model equation is readily shown to be

$$
\mathbf{d}_{n}(\omega)=\boldsymbol{\Phi}_{n} \mathbf{d}_{n-1}(\omega)+\mathbf{w}_{n}(\omega) .
$$

It is important to emphasize that separate sets of statespace equations are formulated for all frequency bins. The innovation noise vector $\mathbf{w}_{n}(\omega)$ is an i.i.d. process, with zeromean and covariance matrix

$$
\mathbb{E}\left\{\mathbf{w}_{n}(\omega) \mathbf{w}_{m}^{H}(\omega)\right\} \triangleq \mathbf{Q}_{n}(\omega) \delta_{m, n} .
$$

Assume that uncorrelated antennas $\mathbf{Q}_{n}(\omega)$ are a diagonal matrix. Following the assumption that the driving noise signals $w^{i j}(\ell, n)$ are uncorrelated for all $\ell$, it is easily shown that

$$
\mathbb{E}\left\{\left|w^{i j}(\omega, n)\right|^{2}\right\}=\sum_{l=0}^{L-1} \mathbb{E}\left\{\left|w^{i j}(\ell, n)\right|^{2}\right\} .
$$

The state-space formulation in the frequency domain is also summarized in Algorithm2.

\subsubsection{Tracking the Singular Vectors of the Bidirectional MIMO} System. A state-space formulation for the singular vectors of the channel matrix [the matrices $\mathbf{U}_{n}(\omega)$ and $\mathbf{V}_{n}(\omega)$ ] is now stated. Several modifications to the model in Section 3.2.1 are required for this exposition. We describe the model for the downlink channel. The model for the uplink is derived in a similar fashion.

Simplifying the channel-dependent transition factors $\alpha^{i j}$ to channel-independent transition factors $\alpha$, the channel matrix is reduced to $\Phi_{n}=\alpha \mathbf{I}$. Then the channel model simplifies to

$$
\mathbf{H}_{n}(\omega)=\alpha \mathbf{H}_{n-1}(\omega)+\mathbf{W}_{n}(\omega) .
$$

The matrix $\mathbf{H}_{n}(\omega)$ was defined in (12), and the innovation noise $\mathbf{W}_{n}(\omega)$ is given by

$$
\mathbf{W}_{n}(\omega) \triangleq\left[\begin{array}{ccc}
w^{11}(\omega, n) & \cdots & w^{N_{t} 1}(\omega, n) \\
\vdots & \vdots & \\
w^{1 N_{r}}(\omega, n) & \cdots & w^{N_{t} N_{r}}(\omega, n)
\end{array}\right] .
$$

For the sake of brevity, we omit the frequency bin index $\omega$ from the following derivation. Using (17), we have

$$
\begin{aligned}
\overline{\mathbf{y}}_{n} & =\mathbf{U}_{n} \mathbf{S}_{n} \mathbf{V}_{n}^{H} \overline{\mathbf{x}}_{n}+\mathbf{z}_{n} \\
& =\mathbf{U}_{n} \mathbf{S}_{n} \mathbf{V}_{n}^{H} \mathbf{V}_{n} \mathbf{x}_{n}+\mathbf{z}_{n} \\
& =\mathbf{U}_{n} \mathbf{S}_{n} \mathbf{x}_{n}+\mathbf{z}_{n} .
\end{aligned}
$$

The last equation can be rewritten as

$$
\overline{\mathbf{y}}_{n}^{T}=\mathbf{x}_{n}^{T}\left(\mathbf{U}_{n} \mathbf{S}_{n}\right)^{T}+\mathbf{z}_{n}^{T} .
$$

Using the $\operatorname{vec}(\cdot)$ operator, we have

$$
\begin{aligned}
\overline{\mathbf{y}}_{n} & =\operatorname{vec}\left(\mathbf{x}_{n}^{T}\left(\mathbf{U}_{n} \mathbf{S}_{n}\right)^{T}\right)+\mathbf{z}_{n} \\
& =\operatorname{vec}\left(\mathbf{x}_{n}^{T}\left(\mathbf{U}_{n} \mathbf{S}_{n}\right)^{T} \mathbf{I}_{N_{r}}\right)+\mathbf{z}_{n} .
\end{aligned}
$$

Using (26), we finally have

$$
\overline{\mathbf{y}}_{n}=\left(\mathbf{I}_{N_{r}} \otimes \mathbf{x}_{n}^{T}\right) \operatorname{vec}\left(\left(\mathbf{U}_{n} \mathbf{S}_{n}\right)^{T}\right)+\mathbf{z}_{n} .
$$

Equation (39) constitutes the measurement equation for the bidirectional tracking scenario with the state vector defined as $\mathbf{d}_{n} \triangleq \operatorname{vec}\left(\left(\mathbf{U}_{n} \mathbf{S}_{n}\right)^{T}\right)$ and $\mathbf{G}_{n}=\left(\mathbf{I}_{N_{r}} \otimes \mathbf{x}_{n}^{T}\right)$. The state equation can be derived from (34) by using the SVD:

$$
\mathbf{U}_{n} \mathbf{S}_{n} \mathbf{V}_{n}^{H}=\alpha \mathbf{U}_{n-1} \mathbf{S}_{n-1} \mathbf{V}_{n-1}^{H}+\mathbf{W}_{n} .
$$

Multiplying both sides of the equation by $\mathbf{V}_{n}$, we get

$$
\mathbf{U}_{n} \mathbf{S}_{n}=\alpha \mathbf{U}_{n-1} \mathbf{S}_{n-1} \mathbf{V}_{n-1}^{H} \mathbf{V}_{n}+\mathbf{W}_{n} \mathbf{V}_{n} .
$$

Using $\mathbf{d}_{n}$ definition yields

$$
\begin{aligned}
\mathbf{d}_{n} & =\operatorname{vec}\left(\left(\mathbf{U}_{n} \mathbf{S}_{n}\right)^{T}\right) \\
& =\operatorname{vec}\left(\left(\alpha \mathbf{U}_{n-1} \mathbf{S}_{n-1} \mathbf{V}_{n-1}^{H} \mathbf{V}_{n}\right)^{T}+\left(\mathbf{W}_{n} \mathbf{V}_{n}\right)^{T}\right) \\
& =\operatorname{vec}\left(\alpha\left(\mathbf{V}_{n-1}^{H} \mathbf{V}_{n}\right)^{T}\left(\mathbf{U}_{n-1} \mathbf{S}_{n-1}\right)^{T}\right)+\operatorname{vec}\left(\left(\mathbf{W}_{n} \mathbf{V}_{n}\right)^{T}\right) \\
& =\operatorname{vec}\left(\left(\mathbf{V}_{n-1}^{H} \mathbf{V}_{n}\right)^{T}\left(\mathbf{U}_{n-1} \mathbf{S}_{n-1}\right)^{T} \alpha \mathbf{I}_{N_{r}}\right)+\operatorname{vec}\left(\left(\mathbf{W}_{n} \mathbf{V}_{n}\right)^{T}\right)
\end{aligned}
$$

Using (26) again, we finally get

$$
\mathbf{d}_{n}=\left(\left(\alpha \mathbf{I}_{N_{r}}\right) \bigotimes\left(\mathbf{V}_{n-1}^{H} \mathbf{V}_{n}\right)^{T}\right) \mathbf{d}_{n-1}+\operatorname{vec}\left(\left(\mathbf{W}_{n} \mathbf{V}_{n}\right)^{T}\right) .
$$

Therefore, the transition matrix is evidently given by $\boldsymbol{\Phi}_{n}=$ $\left(\left(\alpha \mathbf{I}_{N_{r}}\right) \otimes\left(\mathbf{V}_{n-1}^{H} \mathbf{V}_{n}\right)^{T}\right)$. Note that the transition matrix in this case is time varying, due to the term $\mathbf{V}_{n-1}^{H} \mathbf{V}_{n}$, assumed to be known in the application of the Kalman filter in Appendix A. Moreover, it is also assumed that the transmitted signal $\overline{\mathbf{x}}_{n}=\mathbf{V}_{n} \mathbf{x}_{n}$ is accurately calculated. As a consequence, deriving the benchmark for tracking the singular vectors at the receiving side of the subscriber site, that is $\mathbf{d}_{n}=$ $\operatorname{vec}\left(\left(\mathbf{U}_{n} \mathbf{S}_{n}\right)^{T}\right)=\operatorname{vec}\left(\boldsymbol{S}_{n} \mathbf{U}_{n}^{T}\right)$, requires exact knowledge of the singular vectors of the respective transmitting side $\mathbf{V}_{n}$. Note, that the innovation noise covariance matrix, defined in (32), remains intact due to the orthogonality of the matrix $\mathbf{V}_{n}$. For the derivation presented in this paper, we assume that the singular values matrix $\mathbf{S}_{n}$ can be accurately estimated. Singular value estimation is out of the scope of this paper. It should be noted though that decision-directed schemes, which use the predefined constellation, can be applied to estimate the singular values. Finally, at the receiving side of the base station, the state vector is defined as $\mathbf{d}_{n}=$ $\operatorname{vec}\left(\mathbf{S}_{n} \mathbf{V}_{n}^{H}\right)$. The tracking algorithm for the bidirectional communication is depicted in Algorithm 2. 


\section{The Application of the PASTd Algorithm}

Bidirectional MIMO communication channel can be transformed to parallel independent AWGN channels as shown in (17) and in Figure 1. Knowing the right-hand singular vector matrix $\mathbf{V}_{n}$ at the base station site and the left-hand singular vector matrix $\mathbf{U}_{n}$ at the subscriber site suffices for diagonalizing the MIMO channel in both directions. Hence, reliable communication necessitates the calculation of the SVD in both terminals. As, in general, the channel matrix $\mathbf{H}_{n}$ is time varying, direct calculation of the SVD is cumbersome and imposes a computational burden that is too high for practical applications. We therefore propose in this section an efficient method for tracking the SVD matrices. We will show that the received data on each terminal suffice for estimating the corresponding matrix, and hence no feedback between terminals is necessary.

We begin by assuming that the transmitted signals, in both directions, are uncorrelated, white, zero-mean, Gaussian with equal constant power per singular mode:

$$
\mathbb{E}\left(\mathbf{x}_{n} \mathbf{x}_{m}^{H}\right)=\rho \mathbf{I}_{N_{t}} \delta_{m, n}, \quad \mathbb{E}\left(\tilde{\mathbf{x}}_{n} \widetilde{\mathbf{x}}_{m}^{H}\right)=\tilde{\rho} \mathbf{I}_{N_{r}} \delta_{m, n},
$$

where $\mathbb{E}$ denotes the expectation operation, $\rho, \tilde{\rho}$ are the input signals power at the downlink and uplink, respectively, $\mathbf{I}_{N_{t}}$ and $\mathbf{I}_{N_{r}}$ are, respectively, $N_{t} \times N_{t}$ and $N_{r} \times N_{r}$ identity matrices, and $\delta_{m, n}$ is the Kronecker delta. The number of available communication channels is $\min \left(N_{t}, N_{r}\right)$.

We concentrate on describing the tracking procedure for $\mathbf{U}_{n}$ held in the receiving terminal of the subscriber site. The tracking procedure for $\mathbf{V}_{n}$ at the base station is equivalent. Assume the receiver at the base station site, already converged that is, $\mathbf{V}_{n}$ is known. This approximation can be justified by using a proper initialization. Initialization schemes are out of the scope of the current contribution. However, we note by passing that a training sequence can be used while the algorithm is in its acquisition stage. After the channel has been acquired, channel tracking based on the proposed method can be applied. As shown in (17), the received signal at the subscriber site is given by

$$
\overline{\mathbf{y}}_{n}=\mathbf{H}_{n} \overline{\mathbf{x}}_{n}+\mathbf{z}_{n} .
$$

Assuming that the receiver noise is spatially white with unit gain, that is, $\mathbb{E}\left(\mathbf{z}_{n} \mathbf{z}_{m}^{H}\right)=\mathbf{I}_{N_{r}}$ and using (17), the correlation matrix of the vector of received signals is given by

$$
\begin{aligned}
\mathbf{R}_{n}^{\bar{y}} & \triangleq \mathbb{E}\left(\overline{\mathbf{y}}_{n} \overline{\mathbf{y}}_{n}^{H}\right)=\mathbf{H}_{n} \mathbb{E}\left(\overline{\mathbf{x}}_{n} \overline{\mathbf{x}}_{n}^{H}\right) \mathbf{H}_{n}^{H}+\mathbf{I}_{N_{r}} \\
& =\rho \mathbf{H}_{n} \mathbf{V}_{n} \mathbf{V}_{n}^{H} \mathbf{H}_{n}^{H}+\mathbf{I}_{N_{r}}=\mathbf{U}_{n}\left(\rho \mathbf{S}_{n} \mathbf{S}_{n}^{H}+\mathbf{I}_{N_{r}}\right) \mathbf{U}_{n}^{H},
\end{aligned}
$$

where due to the noise power definition, $\rho$ is now the SNR. We conclude that the left singular vectors of $\mathbf{H}_{n}$ are the eigenvectors of $\mathbf{R}_{n}^{\bar{y}}$. Therefore, calculation of the left SVD matrix of $\mathbf{H}_{n}$ can be obtained by calculating the eigenvectors of $\mathbf{R}_{n}^{\bar{y}}$ from the received data vectors $\overline{\mathbf{y}}_{n}$.

The PAST algorithm [15] provides an efficient recursive method for estimating part of (or all) $\mathbf{R}_{n}^{\bar{y}}$ eigenvectors. The method is based on alternative interpretation of the subspace, spanned by several eigenvectors, as a solution to a minimization problem. The minimization of the resulting cost function is then solved by applying the recursive least squares (RLS) technique, combined with a subspace projection approximation. However, usually the obtained basis is not orthogonal. Therefore, we will use a variant of PAST, denoted PASTd, as it uses a deflation technique in order to provide the required orthonormal basis. The main advantage of using the PASTd algorithm is its ability to converge to an orthonormal basis of the correlation matrix and to track its variations.

The algorithm used at the receiver of the subscriber site is summarized in Algorithm 1. We assume that the system exploits all available $\min \left(N_{t}, N_{r}\right)$ channels for the communication. In this summary, we used the following definitions. The eigenvectors comprising the matrix $\mathbf{U}_{n}$ are given by

$$
\mathbf{U}_{n}=\left[\begin{array}{llll}
\mathbf{u}_{n}^{1} & \mathbf{u}_{n}^{2} & \cdots & \mathbf{u}_{n}^{N_{r}}
\end{array}\right] .
$$

$0<\beta \leq 1$ is the forgetting factor of the recursive scheme, and $\hat{\mathbf{u}}_{0}^{i} ; 1 \leq n \leq \min \left(N_{t}, N_{r}\right)$ are the initial estimate of the relevant eigenvectors at time 0 . Note that the initialization set, defined in Algorithm 1, is an orthonormal set.

Similarly, the estimates $\hat{v}_{n}^{j} ; 1 \leq j \leq \min \left(N_{t}, N_{r}\right)$ comprising the right singular value matrix of the channel are estimated at the receiver of the base station site, using the data received from the reverse channel, $\overline{\tilde{\mathbf{y}}}_{n}$. The estimates $\hat{\mathbf{U}}_{n}$ and $\hat{\mathbf{V}}_{n}$ are then used for transmission and reception in both terminals. Recall that $\mathbf{U}_{n}$ is only required at the base station site, while $\mathbf{V}_{n}$ is only required at the subscriber site. Hence, the effective channel is given by

$$
\widehat{\mathbf{S}}_{n}=\hat{\mathbf{U}}_{n}^{H} \mathbf{U}_{n} \mathbf{S}_{n} \mathbf{V}_{n}^{H} \hat{\mathbf{V}}_{n}
$$

which results in a diagonal channel [as in (17)] only had the estimate been exact. In practical scenarios, $\hat{\mathbf{S}}_{n}$ only approximates a diagonal channel.

\section{Performance Measures for Approximate SVD}

In this section, we suggest some information-theoretic-based performance measures, in order to evaluate the quality of the effective channel induced by an approximate SVD procedure (as we are interested in evaluating the proposed PASTdbased algorithm only the frequency-domain formulation is considered here). Arguably, the best such measure is channel capacity; however, the exact computation of the capacity for fading channels is a cumbersome task even for simple block-i.i.d. fading models [22]. Instead, we shall consider the maximum rate that can be achieved under a number of assumptions that simplify both the expression for the achievable rate and the structure of the receiver. We will assume that the fading coefficients are known at the receiver and limit ourselves to coding schemes that first try to diagonalize the channel via an (approximate) SVD and then use each (approximated) mode as a scalar channel. Hence, the residual crosstalk terms due to imperfect diagonalization are not compensated for by joint decoding, capturing the 
negative effect of the approximation. For further simplicity, we shall also assume that the transmitted power is equally divided between the (approximated) modes, without taking into consideration possible difference between channel modes. It should be emphasized that the resulting measures provide only a rough estimate of the rate and are mostly intended as a common yardstick rendering different tracking algorithms commensurable. In what follows, we assume that the process $\left\{\mathbf{H}_{n}\right\}_{n=1}^{\infty}$ is stationary and ergodic, so the suggested performance measures can be estimated arbitrarily well in practice by replacing the expectation with averaging. Note that although we retain the time subscript, all our expressions will in fact depend on the first-order statistics of the channel matrix only.

We first consider the case of perfect SVD. In this case, the maximal achievable rate (with equal mode power and fading known at the receiver) is achieved by a circular symmetric white Gaussian codebook [23] and is given by

$$
\begin{aligned}
R_{\max } & =\mathbb{E} \log \operatorname{det}\left(\mathbf{I}_{N_{t}}+\rho \mathbf{H}_{n} \mathbf{H}_{n}^{H}\right) \\
& =\mathbb{E} \log \operatorname{det}\left(\mathbf{I}_{N_{t}}+\rho \mathbf{S}_{n} \mathbf{S}_{n}^{H}\right) .
\end{aligned}
$$

Note that only the first-order statistics of $H_{n}$ is required, since the channel is memoryless given the fading coefficients, and the latter are assumed independent of the input and known at the reciever. Let us turn now to the case where only an approximate SVD has been performed, via some tracking algorithm. Since the receiver is assumed to decode each of the approximated modes separately, we have two sources of noise: (1) a Gaussian additive noise and (2) crosstalk noise from other modes due to off-diagonal elements. Define the three matrices

$$
\begin{aligned}
& \boldsymbol{\Lambda}_{n} \triangleq \operatorname{diag}\left(\hat{\mathbf{U}}_{n}^{H} \hat{\mathbf{U}}_{n}\right), \\
& \boldsymbol{\Gamma}_{n} \triangleq \operatorname{diag}\left(\hat{\mathbf{S}}_{n} \hat{\mathbf{S}}_{n}^{H}\right)-\operatorname{diag}\left(\widehat{\mathbf{S}}_{n}\right) \operatorname{diag}\left(\hat{\mathbf{S}}_{n}\right)^{H}, \\
& \boldsymbol{\Psi}_{n} \triangleq \operatorname{diag}\left(\hat{\mathbf{S}}_{n}\right) \operatorname{diag}\left(\hat{\mathbf{S}}_{n}\right)^{H},
\end{aligned}
$$

where $\operatorname{diag}(\mathbf{M})$ is defined as a square diagonal matrix with the same diagonal elements as $\mathbf{M}$.

The signal power is given by the square absolute value of the diagonal elements of the matrix $\widehat{\mathbf{S}}_{n}$ multiplied by the perantenna $\operatorname{SNR} \rho$, which is the diagonal of the matrix $\rho \Psi_{n}$. The channel noise is not white due to the fact that the estimate $\hat{\mathbf{U}}_{n}$ is not orthonormal (although it is approximately so). The noise covariance matrix is therefore given by $\hat{\mathbf{U}}_{n}^{H} \hat{\mathbf{U}}_{n}$, and since the receiver performs separate decoding for each mode, we are only concerned with the noise power which is given by the diagonal of $\boldsymbol{\Lambda}_{n}$. The crosstalk noise stems from the off-diagonal elements of the effective channel matrix $\hat{\mathbf{S}}_{n}$. The crosstalk noise power per mode is given by the diagonal elements of the matrix $\hat{\mathbf{S}}_{n}^{H} \widehat{\mathbf{S}}_{n}$, multiplied by the SNR $\rho$. Therefore, the total crosstalk noise power per mode is given by the elements of the diagonal matrix $\rho \boldsymbol{\Gamma}_{n}$, and the total noise per mode is given by the elements of the diagonal matrix $\boldsymbol{\Lambda}_{n}+\rho \boldsymbol{\Gamma}_{n}$. Thus, by using a circular symmetric

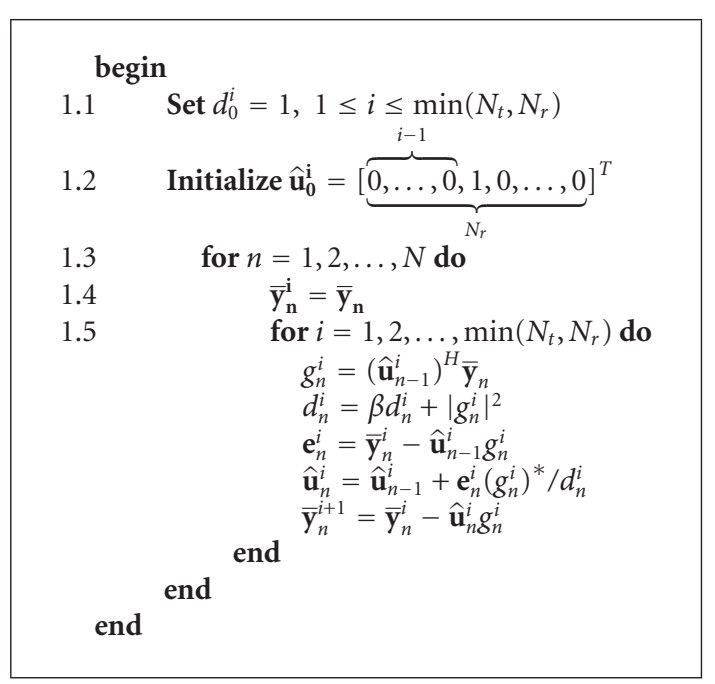

Algorithm 1: Summary of the PASTd algorithm performed at the receiver of the subscriber site (downlink direction).

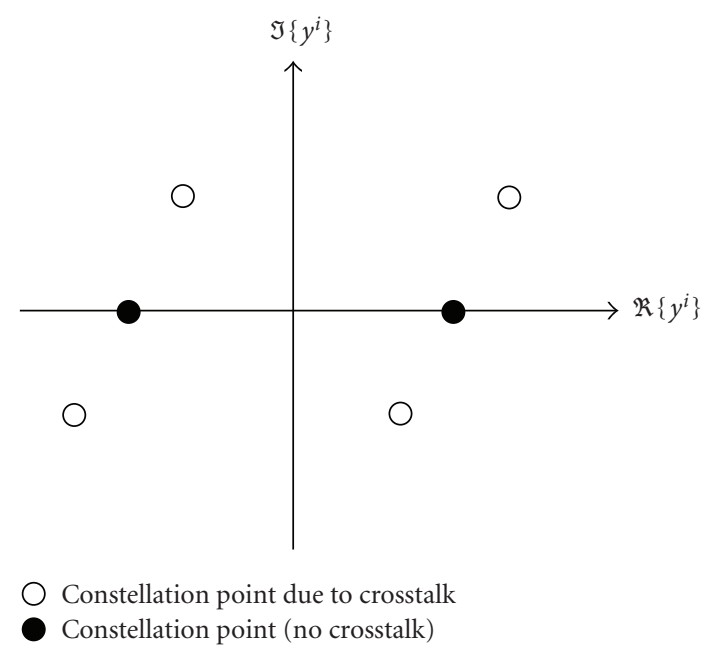

FIGURE 2: Inversion probability in MIMO channel using BPSK signaling.

white Gaussian codebook over the approximated modes and treating the crosstalk as noise, it is easily observed that the following rate is achievable:

$$
\begin{aligned}
R_{\mathrm{eff}} & =\mathbb{E} \log \operatorname{det}\left(\mathbf{I}_{N_{t}}+\rho \boldsymbol{\Psi}_{n}\left(\boldsymbol{\Lambda}_{n}+\rho \boldsymbol{\Gamma}_{n}\right)^{-1}\right) \\
& =\sum_{i} \mathbb{E} \log \left(1+\operatorname{SINR}_{i}\right)
\end{aligned}
$$

where $\operatorname{SINR}_{i}=\rho \psi_{n, i} /\left(\lambda_{n, i}+\rho \gamma_{n, i}\right)$ and $\psi_{n, i}, \lambda_{n, i}$, and $\gamma_{n, i}$ are the diagonal elements of $\boldsymbol{\Psi}_{n}, \boldsymbol{\Lambda}_{n}$, and $\boldsymbol{\Gamma}_{n}$, respectively. Note, that the expectation is taken w.r.t. the random $\operatorname{SINR}_{i}$, which is assumed known at the receiver. Comparing $R_{\text {eff }}$ to $R_{\max }$ can give some idea of how large is the loss in rate due to tracking misalignment.

It is also our aim to explore the degradation incurred when using practical signaling constellation (rather than a Gaussian codebook). For that purpose, we shall restrict 
our attention to the following simple transmission scheme. BPSK signaling $\{-\sqrt{\rho}, \sqrt{\rho}\}$ is transmitted over each of the approximated modes, while a hard decision is taken with respect to that constellation at the receiving side. Thus, for any instantiation of the channel matrix, we essentially have a binary symmetric channel (BSC) over each mode in each direction. The capacity of a BSC is given by $1-h_{b}(p)$, where $p$ is the associated inversion probability and $h_{b}(\cdot)$ is the binary entropy function. If the inversion probabilities constitute a stationary and ergodic process $\left\{p_{n}\right\}_{n=1}^{\infty}$, independent of the input and known at the receiver, then the capacity becomes $\mathbb{E}\left(1-h_{b}\left(p_{n}\right)\right)$. Recall that the inversion probability for BPSK signaling over an AWGN channel with output SNR $\rho_{\text {out }}$ is given by $Q\left(\sqrt{\rho_{\text {out }}}\right)\left(Q(x)=1 / \sqrt{2 \pi} \int_{x}^{\infty} e^{-x^{2} / 2}\right.$ is the Gaussian $Q$-function.). Following this, the maximal achievable rate for BPSK signaling with equal power allocation over the modes and perfect SVD is given by

$$
R_{\max }^{\mathrm{BPSK}}=\sum_{k=1}^{\min \left(N_{r}, N_{t}\right)} \mathbb{E}\left(1-h_{b}\left(Q\left(\sqrt{\rho} s_{n}^{k}\right)\right)\right),
$$

where $s_{n}^{k}$ are the singular values of $\mathbf{H}_{n}$, that is, the diagonal elements of $\mathbf{S}_{n}$, which are assumed known at the receiver.

Turning back to the case of approximate SVD, we will assume for simplicity that the phase of the diagonal elements of the effective channel matrix $\widehat{\mathbf{S}}_{n}$ can be accurately estimated at the receiver, by applying some decision-directed rule. The receiver is then assumed to compensate for the phase, so that the effective channel matrix becomes

$$
\overline{\mathbf{S}}_{n}=\measuredangle\left(\operatorname{diag}\left(\hat{\mathbf{S}}_{n}^{H}\right)\right) \cdot \widehat{\mathbf{S}}_{n}
$$

where $\measuredangle\left(r e^{j \theta}\right) \triangleq e^{j \theta}$ for $r>0$ is applied elementwise. Now, for any transmitted vector from the BPSK constellation, we can find the probability of inversion for each of the BSC's over the approximated modes and then average over all possible transmitted vectors. As in the Gaussian input case, the inversion probability is an accumulated impact of channel noise and crosstalk interference (see Figure 2). It is easy to see that the covariance matrix $\boldsymbol{\Sigma}_{n}$ of the noise after both $\hat{\mathbf{U}}_{n}$ and the phase compensation are applied satisfies

$$
\boldsymbol{\Sigma}_{n}=\measuredangle\left(\operatorname{diag}\left(\hat{\mathbf{S}}_{n}^{H}\right)\right) \cdot \hat{\mathbf{U}}_{n}^{H} \hat{\mathbf{U}}_{n} \cdot \measuredangle\left(\operatorname{diag}\left(\widehat{\mathbf{S}}_{n}\right)\right) .
$$

Note that $\operatorname{diag}\left(\boldsymbol{\Sigma}_{n}\right)=\boldsymbol{\Lambda}_{n}$, that is, $\boldsymbol{\Sigma}_{n}$, has the same diagonal elements as $\boldsymbol{\Lambda}_{n}$. Hence, the noise power per approximated mode remains the same after phase compensation.

Recall that the transmitted vector $\mathbf{x}_{n}$ is of the form $\sqrt{\rho} \mathbf{b}$ for some $\min \left(N_{r}, N_{t}\right)$-dimensional vector $\mathbf{b}$ with elements in $\{1,-1\}$. The corresponding received signal (without the noise) after phase compensation is $\rho \overline{\mathbf{S}}_{n} \mathbf{b}$, and hard-decision decoding is applied only to its real part. Following that, and using a uniform codebook independent over the modes, the following rate is achievable:

$$
R_{\mathrm{eff}}^{\mathrm{BPSK}}=\sum_{k=1}^{\min \left(N_{r}, N_{t}\right)} \mathbb{E}\left(1-h_{b}\left(p_{n}^{k}\right)\right) .
$$

The expectation is taken w.r.t.

$$
\mathbf{p}_{n} \triangleq 2^{-\min \left(N_{r}, N_{t}\right)} \sum_{\mathbf{b}} Q\left(-\Lambda_{n}^{-1} \cdot D_{\mathbf{b}} \cdot \mathfrak{R}\left(\sqrt{\rho} \bar{S}_{n} \mathbf{b}\right)\right),
$$

where $\mathbf{p}_{n}=\left[p_{n}^{1}, \ldots, p_{n}^{\min \left(N_{r}, N_{t}\right)}\right]^{T}$, and $p_{n}^{k}$ is the (random) inversion probability associated with the $k$ th approximated mode, at time $n$. The summation is taken over all vectors $\mathbf{b}$ with elements in $\{1,-1\}, D_{\mathbf{b}}$ is a diagonal matrix with the vector $\mathbf{b}$ on the diagonal and $Q(\cdot)$ is applied elementwise. (Note that $\mathbf{p}_{n}$ is averaged over a uniform input, hence we are considering the capacity of a corresponding "average BSC" with $\mathbf{p}_{n}$ known at the receiver.).

The performance measures above assume that the ergodic nature of the channel is exploited, that is, that coding is performed over many channel instances such that the "entire range" of fading behavior is observed. In practice, coding is usually performed over significantly shorter time frames due to delay and complexity constraints, and a notion of an outage probability for a given target rate is called for. Given a transmission rate $R$ and a fixed block size/time frame, the outage probability is defined to be the probability that the empirical channel observed over the block cannot support the rate $R$. For many fading processes, outage is the dominating error event; hence outage probability is error probability de facto. The outage probability can be estimated arbitrarily well by taking enough averages, counting the fraction of blocks in which the average rate (according to any one of the desired measures above) falls below the target rate $R$.

\section{Experimental Study}

This section is dedicated to performance evaluation of the proposed PASTd-based tracking algorithm. The tracking ability, the effective rate, the outage probability, and SER versus SNR curves are used to evaluate the algorithm and to compare it with the benchmark method based on the Kalman filter as described in Appendix A and the BIMA algorithm, a channel tracking algorithm based on the iterative power method that was proposed by Dahl et al. [12].

6.1. Test Scenario. Simulations were performed for a $2 \times 2$ MIMO channel, that is, $N_{r}=N_{t}=2$. We use a simplified version of Saleh and Valenzuela's [19] model and describe the transfer function of each element of the channel matrix by

$$
H_{n}^{i j}(z)=\sum_{k=1}^{3} a_{n}^{(k)} z^{-\tau_{k}}, \quad \tau_{k} \in\{0,1, \ldots, L-1\},
$$

where the reflection coefficients $a_{n}^{(k)}$ are $\mathrm{AR}(1)$ processes and $\tau_{k}$ are their respective delays, randomly chosen in the allowable channel delay spread. Typically, three coefficients suffice to describe a wide range of actual in-door channels. The delays are fixed for each simulation. However, the channel coefficients are time varying

$$
a_{n}^{(k)}=\alpha a_{n-1}^{(k)}+w_{n}^{(k)}, \quad 0<\alpha<1, \quad w_{n}^{(k)} \sim \mathcal{C} \mathcal{N}\left(0, \gamma^{-2 k}\right) .
$$


The channel coefficients variance is obeying an exponential decay profile. The reflection coefficients were selected independently for each matrix element $1 \leq i, j \leq 2$.

The symbol rate was set to $20 \mathrm{MHz}$, and the maximal delay spread is set to $L=16$ symbols $(0.8 \mu \mathrm{s})$. The profile parameter $\gamma$ is set to $3 \mathrm{~dB}$. This setting was converted into a 64-subcarrier OFDM-MIMO setting, using a 16symbol cyclic prefix, for the comparison of time- versus frequency-domain methods. In the rest of the evaluation, 32 subcarriers and the same cyclic prefix length were used. The AR parameter was set to $\alpha=1-10^{-4}$ which corresponds to a coherence time of $\sim 10^{4}$ OFDM symbols $(0.5 \mathrm{~ms})$. Since normally it is not possible for the uplink and downlink to transmit simultaneously under the reciprocity model, it is more realistic to assume a TDD scheme. The TDD packet size was set to 100 OFDM symbols $(5 \mu \mathrm{s})$. Simulations were performed over a time interval of $4 \cdot 10^{4}$ OFDM symbols $(2 \mathrm{~ms})$. We repeated each experiment 100 times and averaged the results. Since the method presented aims at tracking rather than acquisition, the channel matrix estimate was initialized with the correct value. For a fair comparison with the alternative algorithm and the benchmark, both the BIMA algorithm and the Kalman filter were also initialized with the correct value of the channel.

6.2. Test Procedure. Performance evaluation of the proposed method is carried out as follows. First, time-domain tracking schemes are compared with frequency-domain schemes (using the Kalman-based benchmarks) for estimating the expected performance loss due to the use of the latter. It should be stressed that the PASTd tracking scheme is a frequency-domain algorithm. Second, the proposed method is evaluated and compared with the BIMA method and the Kalman benchmark (frequency-domain, bidirectional channel instance) by means of inspecting tracking traces and by calculating the effective communications rate for Gaussian input signals. Finally, the proposed communication scheme is evaluated by estimating the outage probability for both Gaussian and BPSK input signals and the SER versus SNR curves for 4-QAM constellation.

The BIMA technique [12] uses the iterative power method for tracking the right- and left-singular vectors of the channel. Application of the technique necessitates transmission of $b$ symbols, where $b>\min \left(N_{r}, N_{t}\right)$. It is therefore implicitly assumed that the channel is almost constant over $b$ OFDM symbols.

One of the key components of the BIMA method is the slicer, responsible for the BPSK detection. In our implementation, we used a slightly modified version to enable detection of arbitrary constellations. This modification was obtained by replacing the sign operator with a slicer suitable for higher constellations. For a fair comparison between BIMA and the proposed method, both algorithms are using the correct singular values.

Reliable communications can only be guaranteed if the estimated singular vectors in both sites form an orthonormal basis. The deflation procedure in the PASTd technique guarantees approximate orthonormalization of the basis vector. In the BIMA algorithm, the orthonormalization is obtained by sorting the intermediate basis vector according to their norm and then applying QRD.

As a consequence of the orthonormalization step, the computational complexity imposed by both algorithms significantly differs. We only present and compare the number of operations at the receiver side. Let $N$ be either $N_{t}$ or $N_{r}$. In BIMA algorithm, the computational complexity of calculating the norm and then sorting the basis vectors is about $O\left(N^{2}\right)$, and the QRD requires $O\left(N^{3}\right)$ operations. Overall, the number of multiplications per symbol for each OFDM subcarrier is $\left(4 N b+3 N^{2}+3 N^{3}\right) / b$. The computational burden imposed by the PASTd algorithm is $4 N^{2}+3 N$ multiplications per subcarrier which is significantly lower than the complexity of the BIMA algorithm for large number of antennas. The Kalman filter, which assumes known input signal, cannot be used in a realistic scenario and is therefore only used in this paper as a benchmark. For this reason, we do not present the associated computational complexity.

6.3. Channel Tracking. In this section, tracking ability is evaluated. Firstly, we compare time- versus frequency-domain schemes using the Kalman filter benchmark. Secondly, the proposed method is compared with the alternative BIMA tracking algorithm and with the Kalman-based benchmark.

The PASTd-based proposed algorithm is implemented in the frequency-domain. It is therefore beneficial to demonstrate the expected degradation due to the frequency-domain implementation. Define the mean normalized estimation error performance measure (averaged over all channels and all time indexes):

$$
\text { MSE }=\frac{\mathbb{E}\left\{\left\|\hat{\mathbf{d}}_{n \mid n}-\mathbf{d}_{n}\right\|^{2}\right\}}{\mathbb{E}\left\{\left\|\mathbf{d}_{n}\right\|^{2}\right\}},
$$

where $\mathbf{d}_{n}$ is the channel to be tracked. $\mathbf{d}_{n}=\mathbf{h}_{n}$ is given by (3)-(4) for the time-domain algorithm and by (28) for the frequency-domain algorithm. The MSE as a function of the input signal-to-noise-ratio (SNR) is depicted in Figure 3 for both time-domain and frequency-domain Kalman filters. 100 independent runs were averaged to obtain the results in the graph. It is evident that the timedomain Kalman-based tracking algorithm outperforms the frequency-domain Kalman-based tracking algorithm over the entire SNR range. In input SNR levels lower than $20 \mathrm{~dB}$, the difference between the algorithms becomes insignificant.

With this conclusion in mind, we can proceed to the performance evaluation of the proposed algorithm. In Figures 4(a), 4(b), we show tracking traces of the proposed PASTdbased algorithm and compare them to the traces obtained by the BIMA algorithm and the benchmark obtained by the Kalman filter (which utilizes practically unavailable information). The real channel trace and the benchmark obtained by the bidirectional frequency-domain Kalman filter are also depicted in the same graphs. All tracking traces were simulated using $\mathrm{SNR}=30 \mathrm{~dB}$. For the BIMA algorithm, we used $b=5$ as a compromise between tracking ability and reliable estimation. It is evident that the tracking ability of 


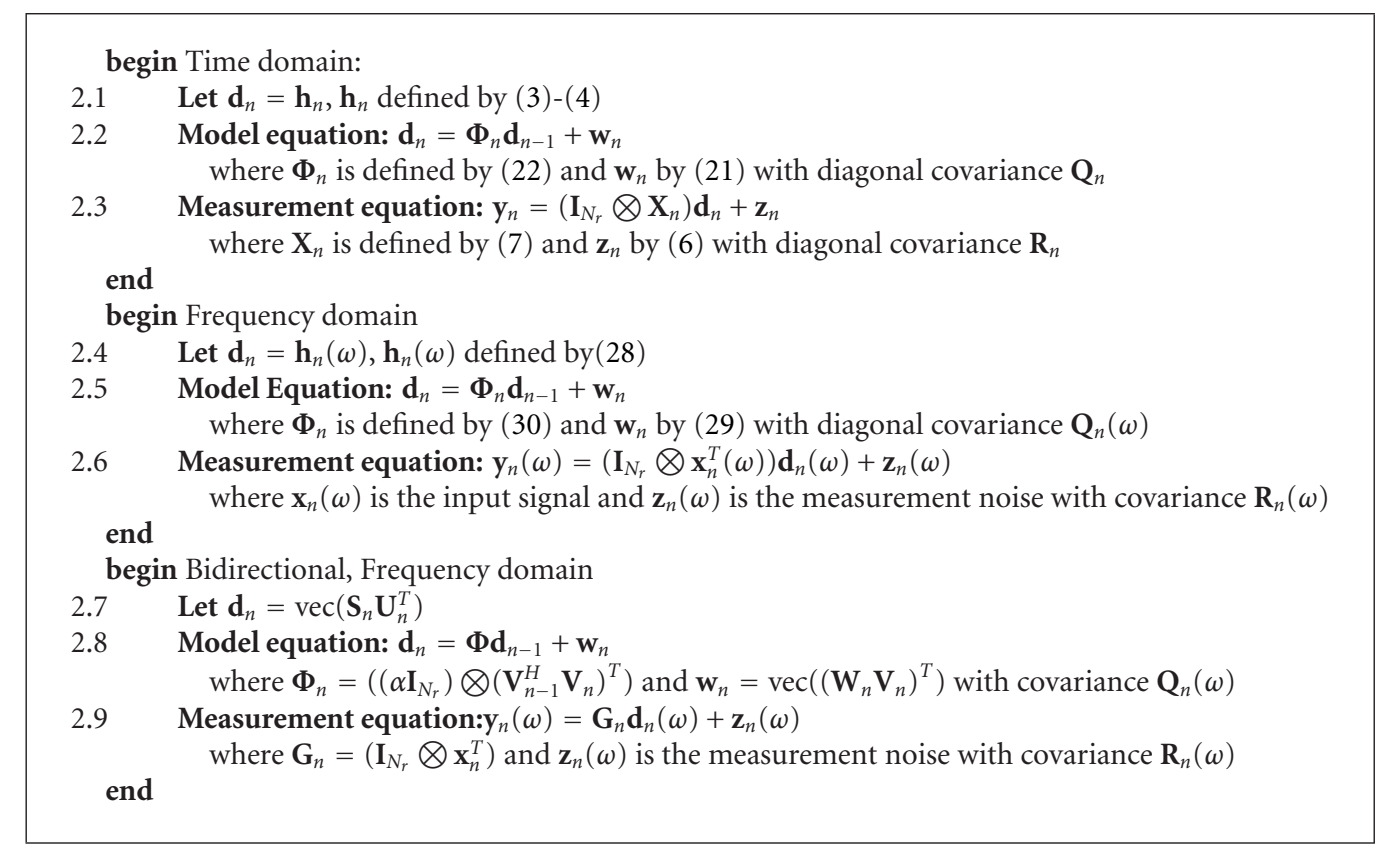

Algorithm 2: State-space formulation at the subscriber site.

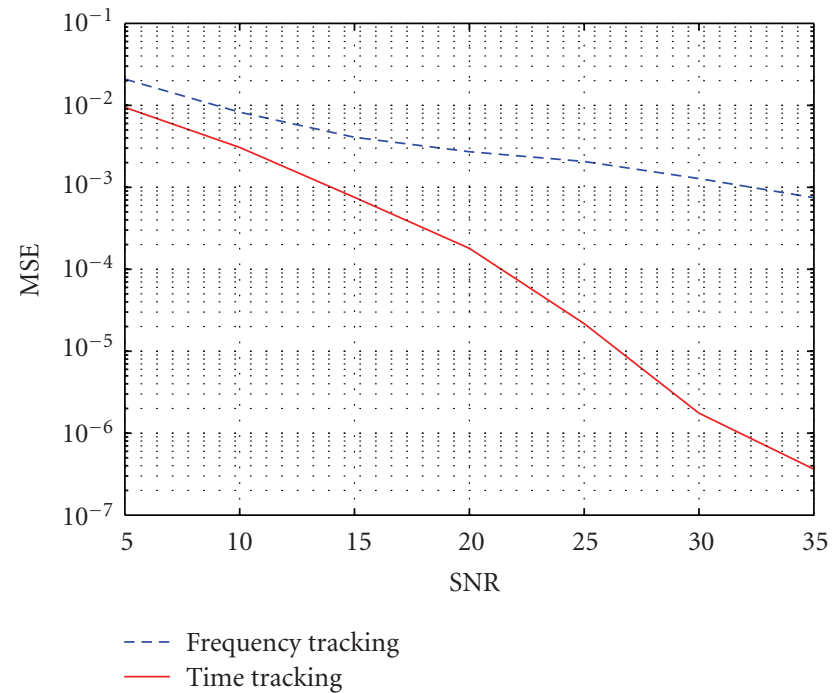

FIgURE 3: MSE versus SNR for time- and frequency-domain Kalman filter-based benchmarks.

the proposed method outperforms the tracking ability of the BIMA algorithm. We conclude that the proposed algorithm has the ability to track rapidly changing MIMO channels.

6.4. Effective Rate, Outage probability, and SER versus SNR. In this section, a communication system based on the proposed method is evaluated. In Figure 5, the effective rate obtained by the PASTd-based communication system with Gaussian input and uniform power loading is depicted. For comparison, the effective rate obtained by the BIMA-based system and the bidirectional frequency-domain Kalman filter benchmark are also depicted and compared with the maximum achievable rate (49), under the uniform power loading assumption.

It is evident that the rate loss due to the tracking ability of the PASTd algorithm is only minor for SNR not exceeding $10 \mathrm{~dB}$.

We proceed by examining the empirical outage probability obtained by the PASTd algorithm for Gaussian input and for BPSK constellation. Simulations were carried out using the optimal parameters for the PASTd algorithm, as explained in [16]. The outage probability was estimated assuming that coding takes place only at the packet level. 100 OFDM symbols $(5 \mu \mathrm{s})$ constitute a packet. The results are depicted in Figure 6(a) for the BPSK input and in Figure 6(b) for Gaussian input. To obtain the results in the graphs, 200 runs, each using 400 packets of 100 OFDM symbols each (i.e., $2 \mathrm{~ms}$ for each run), were used.

As a reference, the effective rate of a communication scheme, based on the PASTd algorithm, is also depicted in the graph. For BPSK constellation, a negligible outage probability can be guaranteed for communication rate of $17.5 \mathrm{Mbit} / \mathrm{sec}$ (compared with the effective rate of 23.7 Mbit/second). For Gaussian input, communicating at a rate of $55.5 \mathrm{Mbit} / \mathrm{second}$ (compared with the effective rate of 64.4 Mbit/second) guarantees very low outage probability.

Finally, SER as a function of SNR is presented in Figure 7 for 4-QAM constellation. In the graph legend, strong and weak modes pertain to high and low singular values, respectively. Recall that uniform power loading per mode was used according to our transmission scheme. It is anticipated that a more sophisticated communication scheme using waterfilling [24] will improve the performance of the proposed communication scheme. 


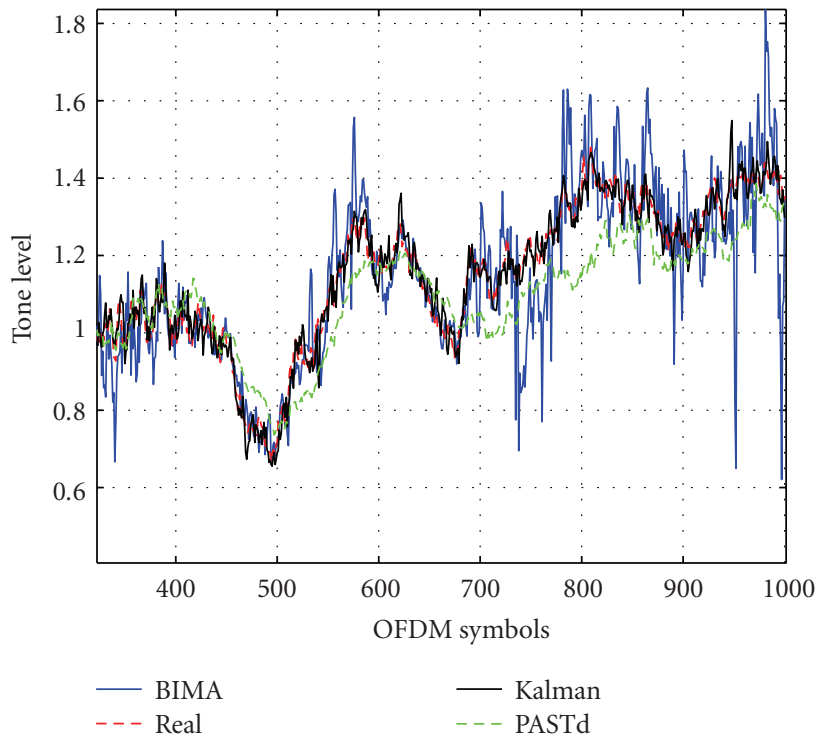

(a) MIMO channel $(1,1)$

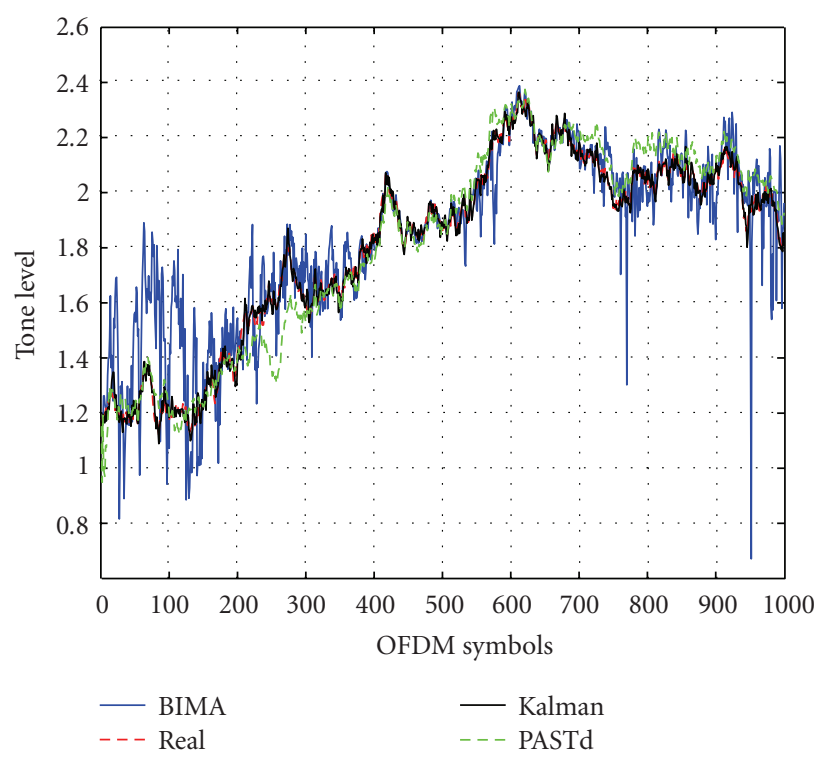

(b) MIMO channel $(2,1)$

FIGURE 4: Tracking traces for OFDM subcarrier number 32 as a function of the symbol index $n$. Comparison between the real, bidirectional frequency-domain benchmark, the BIMA algorithm, and the proposed PASTd-based algorithm.

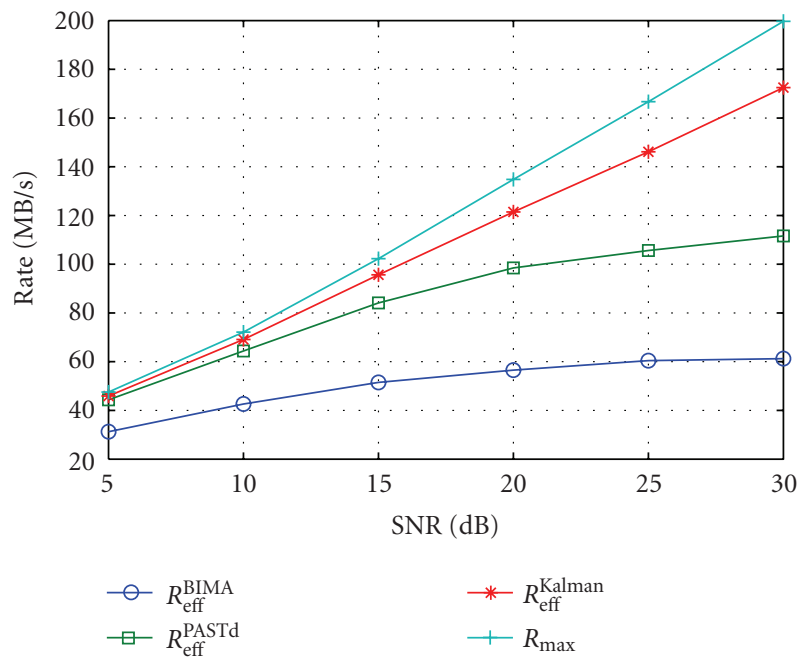

FIGURE 5: Effective rate of the PASTd algorithm compared with the BIMA algorithm, the rate obtained by the Kalman-based benchmark, and the maximal achievable rate obtained by full CSI for uniform power loading transmission. Input signal is Gaussian.

\section{Summary}

In this paper, a novel tracking algorithm for OFDM-MIMO channels in TDD regime was derived. The frequency-domain method uses the PASTd procedure for tracking the SVD of the channel matrix.

The method was compared with the BIMA tracking algorithm, which is based on the power method for calculating the channel singular vectors and with a benchmark derived for this evaluation study. The benchmark is obtained by the application of the Kalman filter to the problem at hand using known input signals and channel statistics. We measure the performance of the various algorithms by evaluating the effective communication rate and the outage probability. It is shown that the proposed algorithm outperforms the BIMA algorithm and exhibits minor performance degradation with respect to the Kalman benchmark (which uses unavailable signal and channel information). Finally, the proposed blind tracking method is evaluated by testing the SER versus SNR for BPSK and 4-QAM constellations.

The PASTd-based algorithm is therefore shown to be a reliable and computationally efficient tracking algorithm for OFDM-MIMO channels.

\section{Appendix}

\section{A. The Application of the Kalman Filter}

The Kalman filter is used in the paper solely as a performance benchmark for our proposed method. Three instances of the Kalman filter are used in the paper. The time-domain and frequency-domain instances are used for roughly estimating the expected performance loss due to the application of the PASTd-based tracking algorithm in the frequency domain. The bidirectional frequency-domain instance of the Kalman filter is used for evaluating the proposed communication scheme (based on either PASTd or BIMA channel tracking algorithms).

The Kalman filter is based on state-space formulation of the system's dynamics. Assume that the underlying process of interest $\mathbf{d}_{n}$ satisfies the following recursive model equation:

$$
\mathbf{d}_{n}=\boldsymbol{\Phi}_{n} \mathbf{d}_{n-1}+\mathbf{w}_{n}
$$




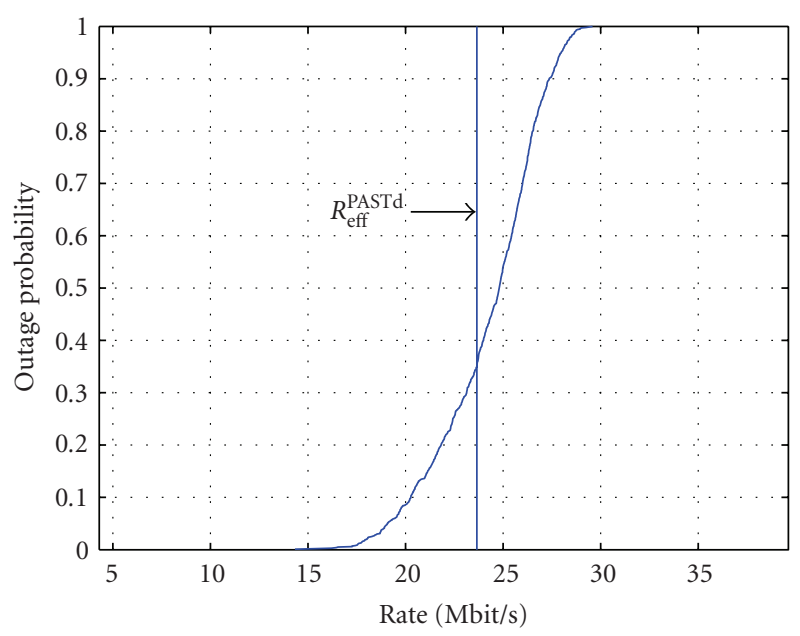

(a) BPSK input (hard decision)

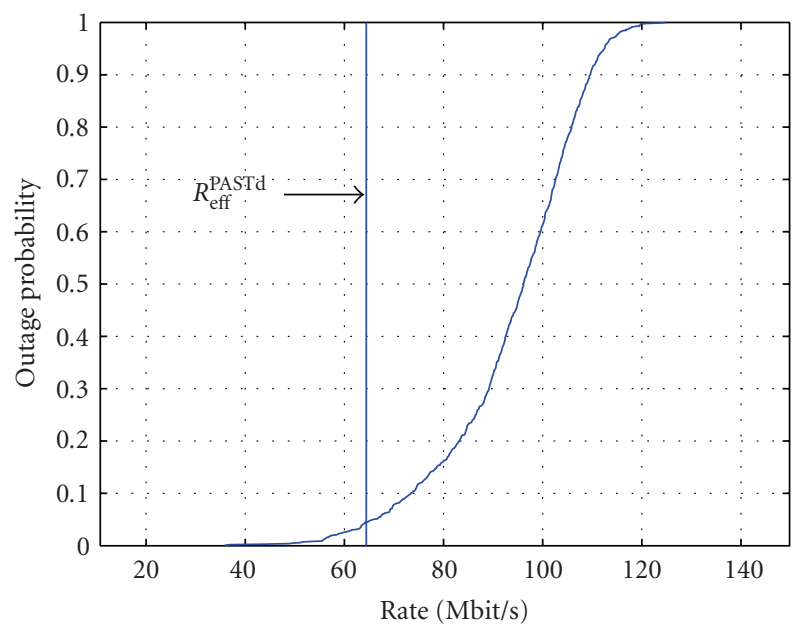

(b) Gaussian input

Figure 6: Rate versus outage, $\mathrm{SNR}=10 \mathrm{~dB}$ per mode.

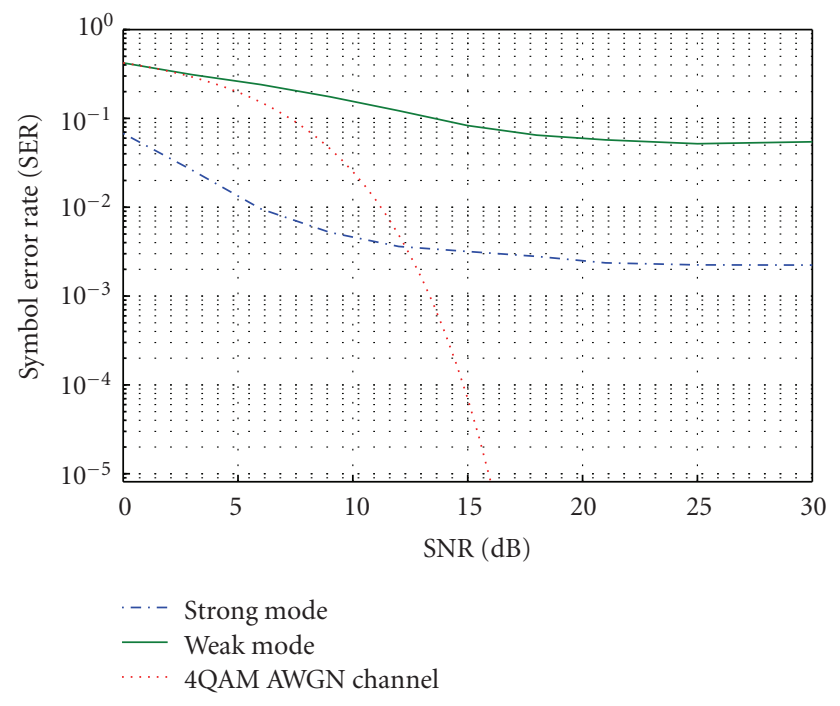

FIgURE 7: SER versus SNR for 4-QAM input.

where $\mathbf{d}_{n}$ is the desired state vector, $\boldsymbol{\Phi}_{n}$ is the transition matrix, and $\mathbf{w}_{n}$ is the innovation process. Further, assume that the measurement $\mathbf{y}_{n}$ is related to the state vector $\mathbf{d}_{n}$ via the following measurement equation:

$$
\mathbf{y}_{n}=\mathbf{G}_{n} \mathbf{d}_{n}+\mathbf{z}_{n}
$$

where $\mathbf{G}_{n}$ is the measurement matrix and $\mathbf{z}_{n}$ is the noise process.

Define $\hat{\mathbf{d}}_{n \mid n}$ as the casual minimum MSE estimator of $\mathbf{d}_{n}$ in (A.1) based on the measurements $\mathbf{y}_{k}, k=1,2, \ldots, n$ in (A.2), and let $\mathbf{P}_{n \mid n}=\mathbb{E}\left\{\left(\hat{\mathbf{d}}_{n \mid n}-\mathbf{d}_{n}\right)\left(\hat{\mathbf{d}}_{n \mid n}-\mathbf{d}_{n}\right)^{H}\right\}$ be its respective error covariance matrix. The Kalman procedure is initialized with $\hat{\mathbf{d}}_{0 \mid 0}=\mathbb{E}\left\{\mathbf{d}_{0}\right\}$, the expectation of the state vector at time instant 0 , and $\mathbf{P}_{0 \mid 0}=\mathbf{P}_{0}$, the respective covariance matrix. We used the correct channel as an initialization, since we are interested in tracking rather than acquisition performance.

The Kalman filter is a recursive procedure [20], in which two estimation stages are applied in each time instant $n=$ $1,2, \ldots$. In the first step, often referred to as the propagation step, $\widehat{\mathbf{d}}_{n \mid n-1}$, the optimal estimate of $\mathbf{d}_{n}$ based on all previous measurements up to $\mathbf{y}_{n-1}$, is calculated. This step, which does not involve the current measurement, predicts the statevector value based on the previous estimate $\hat{\mathbf{d}}_{n-1 \mid n-1}$ and the known dynamics of the process. In the second step, often denoted the update step, the current measurement $\mathbf{y}_{n}$ is utilized to update the estimate $\widehat{\mathbf{d}}_{n \mid n-1}$ from the first step. The update optimally weights the propagated estimate and the innovation embedded in the current measurement. The weighting is given by the Kalman gain matrix $K_{n}$, resulting in an optimal estimate $\hat{\mathbf{d}}_{n \mid n}$ of $\mathbf{d}_{n}$ based on the measurements $\mathbf{y}_{1}, \ldots, \mathbf{y}_{n}$. The algorithm then proceeds to the next time instant $n+1$. For the application of the Kalman filter, $\mathbb{E}\left\{\mathbf{w}_{n} \mathbf{w}_{m}^{H}\right\}=\mathbf{Q}_{n} \delta_{m n}$, the innovation noise covariance matrix, and $\mathbb{E}\left\{\mathbf{z}_{n} \mathbf{z}_{m}^{H}\right\}=\mathbf{R}_{n} \delta_{m n}$, the measurement noise covariance matrix, are assumed to be a priori known.

The Kalman gain $\mathbf{K}_{n}$, calculated by the recursive procedure, is optimally weighting the innovation induced by the measurements and the known dynamics.

The different instances of (A.1) and (A.2) are summarized in Algorithm 2 for time-domain, frequency-domain, and bidirectional frequency-domain tracking schemes.

\section{Acknowledgments}

This research was partially supported by MAGNET ISRC consortium, Ministry of Industry, Trade and Labor, the Government of Israel. 


\section{References}

[1] A. J. Paulraj, D. A. Gore, R. U. Nabar, and H. Bölcskei, "An overview of MIMO communications-a key to gigabit wireless," Proceedings of the IEEE, vol. 92, no. 2, pp. 198-217, 2004.

[2] "IEEE standard for information technology-telecommunications and information exchange between systemslocal and metropolitan area networks-specific requirements_part 11: wireless lan medium access control (MAC) and physical layer (PHY) specifications," IEEE Std 802.11-2007 (Revision of IEEE Std 802.11-1999), pp. C1-1184, 2007.

[3] G. Lebrun, J. Gao, and M. Faulkner, "MIMO transmission over a time-varying channel using SVD," IEEE Transactions on Wireless Communications, vol. 4, no. 2, pp. 757-764, 2005.

[4] C. Komninakis, C. Fragouli, A. H. Sayed, and R. D. Wesel, "Multi-input multi-output fading channel tracking and equalization using Kalman estimation," IEEE Transactions on Signal Processing, vol. 50, no. 5, pp. 1065-1076, 2002.

[5] T. Roman, M. Enescu, and V. Koivunen, "Time-domain method for tracking dispersive channels in MIMO OFDM systems," in Proceedings of the IEEE International Conference on Accoustics, Speech, and Signal Processing (ICASSP '03), pp. 393-396, April 2003.

[6] S. B. Bulumulla, S. A. Kassam, and S. S. Venkatesh, "Adaptive diversity receiver for OFDM in fading channels," in Proceedings of the IEEE International Conference on Communications, vol. 3, pp. 1325-1329, Atlanta, Ga, USA, 1998.

[7] Z. Cheng and D. Dahlhaus, "Time versus frequency domain channel estimation for OFDM systems with antenna arrays," in Proceedings of the 57th Vehicular Technology Conference, vol. 1, pp. 651-655, Jeju, Korea, 2003.

[8] X. Hou, X. Zhao, C. Yin, and G. Yue, "Unified view of channel estimation in MIMO-OFDM systems," in Proceedings of the International Conference on Wireless Communications, Networking and Mobile Computing (WCNM '05), vol. 1, pp. 54-58, September 2005.

[9] R. Haeb-Umbach and M. Bevermeier, "OFDM channel estimation based on combined estimation in time and frequency domain," in Proceedings of the IEEE International Conference on Acoustics, Speech and Signal Processing (ICASSP '07), vol. 3, pp. 277-280, Honolulu, Hawaii, USA, April 2007.

[10] J. Grolleau, E. Grivel, and M. Najim, "Subspace identification method for rayleigh channel estimation," in Proceedings of the IEEE International Conference on Acoustics, Speech and Signal Processing (ICASSP '07), pp. 449-452, April 2007.

[11] T. Dahl, N. Christophersen, and D. Gesbert, "BIMA: blind iterative MIMO algorithm," in 2002 IEEE International Conference on Acoustic, Speech, and Signal Processing (ICASSP '02), vol. 3, pp. 2365-2368, May 2002.

[12] T. Dahl, N. Christophersen, and D. Gesbert, "Blind MIMO eigenmode transmission based on the algebraic power method," IEEE Transactions on Signal Processing, vol. 52, no. 9, pp. 2424-2431, 2004.

[13] T. Dahl, S. S. Pereira, N. Christophersen, and D. Gesbert, "Intrinsic subspace convergence in TDD MIMO communication," IEEE Transactions on Signal Processing, vol. 55, no. 6, pp. 2676-2687, 2007.

[14] S. Barbarossa and A. Scaglione, "Theoretical bounds on the estimation and prediction of multipath time-varying channels," in Proceedings of the IEEE Interntional Conference on Acoustics, Speech, and Signal Processing (ICASSP '00), vol. 5, pp. 2545-2548, June 2000.
[15] B. Yang, "Projection approximation subspace tracking," IEEE Transactions on Signal Processing, vol. 43, no. 1, pp. 95-107, 1995.

[16] S. Gannot, A. Lesnem, O. Shayevitz, and E. Zehavi, “Tracking a MIMO channel singular value decomposition via projection approximation," in Proceedings of the 24th IEEE Convention of Electrical and Electronics Engineers in Israel, pp. 91-94, Eilat, Israel, 2006.

[17] W. Liu, L. L. Yang, and L. Hanzo, "Subspace tracking based blind MIMO transmit preprocessing," in Proceedings of the IEEE 65th Vehicular Technology Conference (VTC '07), pp. 2228-2232, April 2007.

[18] L. Ehrenberg, S. Gannot, A. Leshem, and E. Zehavi, "Performance bounds for channel tracking algorithms for MIMO systems," in Proceedings of the IEEE International Conference on Acoustics, Speech and Signal Processing (ICASSP '08), pp. 3085-3088, April 2008.

[19] A. A. M. Saleh and R. A. Valenzuela, "A statistical model for indoor multipath propagation," IEEE Journal on Selected Areas in Communications, vol. 5, no. 2, pp. 128-137, 1987.

[20] M. S. Grewal and A. P. Andrews, Kalman Filtering, Theory and Practice, Information and System Sciences series, PrenticeHall, NJ, USA, 1993.

[21] K. B. Petersen and M. S. Pedersen, "The matrix cookbook," http://matrixcookbook.com/.

[22] T. L. Marzetta and B. M. Hochwald, "Capacity of a mobile multiple-antenna communication link in rayleigh flat fading," IEEE Transactions on Information Theory, vol. 45, no. 1, pp. 139-157, 1999.

[23] İ.E. Telatar, "Capacity of multi-antenna Gaussian channels," Tech. Rep. Rm. 2C-174, Lucent Technologies, Bell Laboratories, 1995.

[24] W. Yu and J. M. Cioffi, "Constant-power waterfilling: performance bound and low-complexity implementation," IEEE Transactions on Communications, vol. 54, no. 1, pp. 23-28, 2006. 